VI.

Aus dem Laboratorium Sir Victor Horsleys für experimentelle Neurologie im University College in London.

\title{
Zur Technik der experimentellen Untersuchungen am Gehirn, insbesondere am Sehhïgel ${ }_{\text {1) }}^{1}$.
}

\author{
Von \\ Professor B. Pfeifer, \\ Direktor der Landesheilanstalt Nietleben bei Halle a. $\mathrm{S}$. \\ (Mit 2 Textfiguren.)
}

Ein Beitrag zu einer Festschrift für Eduard Hitzig kann zweifellos kein geeigneteres Gebiet betreffen als das der experimentellen Hirnforschung.

Ist es doch Hitzig gewesen, der im Jahre 1870 in einer in Gemeinschaft mit Fritsch veröffentlichten Arbeit „Ueber die elektrische Erregbarkeit des Grosshirns" die heute gültige Lehre von der Funktion der Grosshirnrinde begruindete. Der Nachweis, dass das zentrale Nervensystem und insbesoudere die Grosshirnrinde im Gegensatz zu der bis dahin herrschenden allgemeinen Anschauung in Bezug auf eine so wesentliche allgemeine Eigenschaft wie die Erregbarkeit keinen Unterschied gegenüber dem Verhalten der peripheren Nerven zeigt, war an und für sich schon von höchster Bedeutung. Dazu kam aber ausserdem noch der hohe Wert, der der Entdeckung der elektrischen Reizung als Mittel zur Erforschung der Funktion der Hirnrinde zukommt. Mit Hilfe dieser Methode gelang es vor allem, die elektrisch erregbaren und die elektrisch unerregbaren Anteile der Hirnrinde von einander abzugrenzen und weiterhin im Gebiet der motorisch erregbaren Rinde die feinere Lokalisation der einzelnen Bewegungsformen der verschiedenen Körperregionen zu ermitteln. Hitzig selbst hat bereits in der erwähnten Arbeit eine grosse Fülle von Tatsachen über die durch elektrische Reizung festgestellte cerebrale Innervation der Bewegung beigebracht,

1) Nach einem auf der XVIII. Versammlung der Vereinigung mitteldeutscher Psychiater und Neurologen in Halle gehaltenen Vortrag. 
so dass damit eine vollkommen gesicherte Grundlage geschaffen war. Der weitere Ausbau und die Fortentwickelung der Lehre blieb aber seine eigentliche Lebensarbeit auf wissenschaftlichem Gebiete. In den Jahren 1901-1903, also mehr als 30 Jahre nach der Publikation "Ueber die elektrische Erregbarkeit der Grosshirns" veröffentlichte Hitzig eine Reihe von Arbeiten unter dem Titel „Alte und nene Untersuchungen über das Gehirn". In dem Vorwort zu diesen Arbeiten bemerkt er, dass diese Untersuchungen nach Vollendung der Reorganisation der Landesheilanstalt Nietleben im Jahre 1883 begonnen wurden, dass sie dann eine erste Unterbrechung durch die Gründung der provisorischen Klinik in Halle im Jahre 1885 und eine weitere durch die mit der Gründung und Organisation der definitiven Klinik verbundenen Arbeiten und Aufgaben erfuhren. Die erwähnten Arbeiten Hitzigs erstrecken sich also auch über den Zeitraum, dessen 25 jährige Wiederkehr den Anlass zu der Herausgabe dieser Festschrift bildet.

Für unser Thema ist nun ron besonderem Interesse, welchen Standpunkt Hitzig in diesen Arbeiten bezüglich der Technik der experimentellen Hirnuntersuchungen einnimmt.

Was zunächst die elektrische Untersuchung angeht, so legt Hitzig besonderen Wert darauf, diejenigen Stellen ausfindig zu machen, deren Reizung bei der geringsten, überhaupt wirksamen Stromstärke einen Reizeffekt ergibt. Die Reizeffekte selbst seien besonders daraufhin zu erforschen, $o b$, unter welchen Bedingungen und in welcher Art sie isoliert oder zu gemeinschaftlichen Muskelaktionen kombiniert auftreten. Isolierte Reizerscheinungen kommen nach Hitzig nur bei Anwendung der Reizung mit einzelnen Schlägen des galvanischen Stromes bezw. mit kurzdauernden Induktionsschlägen zustande, während kombinierte Muskelaktionen leichter und vollkommener mit Hilfe von Induktionsströmen von längerer Dauer zu erzielen seien. Die letztere Bewegungsform sei nicht der Effekt der Reizung des eigentlichen Fokus, d. h. der zentralsten und kleinsten Stelle, die überhaupt auf einen Reiz reagiert, sondern beruhe auf einem Uebergang des Reizes auf die Nachbarschaft und auf Summationswirkung. Jede strenge Untersuchung der Hirnrinde miisse sich daher beider Reizmethoden bedienen, der einen zur Aufsuchung der Foci, der anderen zwecks Feststellung der von diesen aus zu erzielenden Bewegungskombinationen. Durch alleinige Anwendung von faradischen Strömen von grosser lntensität und langer Dauer könne das Vorhandensein eines motorischen Innervationszentrums an einer zirkumskripten Stelle der Hirnrinde niemals bewiesen werden. Auf Nichtbeachtung dieser Kautelen von seiten Munks führt Hitzig die zwischen ihm selbst und Munk bestehende Differenz der Untersuchungs- 
resultate bezüglich der elektrischen Erregbarkeit der Stimhirnrinde zurïck.

Ueber die auf Zerstörung bezw. Entfernung von Hirnanteilen hinzielenden Operationsmethoden sagt Hitzig, dass deren verschiedenartige Anwendungsweise hauptsächlich bestimmend gewesen sei für die grundsätzlich verschiedenen Anschauungen über die Funktion der Hirnrinde. Er selbst sei durch den Umstand, dass ganz bestimmte motorische Reizerscheinnngen mit Hilfe des elektrischen Stroms von zirkumskripten Rindenpartien ausgelöst werden können, mit Notwendigkeit dazu gedrängt worden, auch entsprechende Ausfallserscheinungen anzustreben und daher möglichst kleine, den Reizstellen entsprechende Exstirpationen von Rindenteilen vorzunehmen.

Den entgegengesetzten Weg hat Goltz eingeschlagen, indem er möglichst grosse Teile des Gehirns entfernte. Wenn Hitzig auch einerseits den Wert der Ausschaltung grosser Hirnanteile für das Studium gewisser Fragen der Hirnphysiologie anerkannte, so bestritt er doch andererseits deren Bedeutung für die Untersuchung der Lokalisation. der Hirnrindenfunktion. Das grobe Verfahren von Goltz, welcher mittels einer Druckpumpe die zwischen zwei Trepanlöchern befindliche Hirnmasse herauspülte, lasse nicht einmal die Möglichkeit zu, den Eingriff auf den Vorder- oder Hinterlappen des Gehirns zu beschränken. Ausserdem finde eine weit in die Tiefe greifende Zerstörung statt und es komme zu Hirndruckerscheinungen. Die Annahme von Goltz, dass er keinen Unterschied zwischen den Folgeerscheinungen einer Zerstörung: des vorderen und des hinteren Hirnanteils habe feststellen können, sei demnach die notwendige Konsequenz der Art der von ihm vorgenommenen Operationen.

Hitzig gibt dann weiterhin noch eine Reilue von allgemeinen Regeln, die bei der Vornahme von experimentellen Untersuchungen am Gehirn zu befolgen seien. Vor allem sei es erforderlich, dass die einzelnen Versuche in jeder Versuchsreihe gleichwertig seien, und dass sie so beschrieben werden, dass ein Vergleich mit fremden Versuchen durchführbar sei. Die Lokalität müsse sowohl was die angegriffenen Windungen, als die Tiefe des Eingriffs als auch etwaige sekundäre Läsionen angehe, genau beschrieben werden. Auch müssten die einzelnen Versuche von dem gleichen Experimentator unter gleichen Versuchsbedingungen so oft wiederholt werden, bis ein zweifelloses und konstantes Resultat erzielt sei.

Bei den experimentellen Hirnforschungen Hitzig's handelte es sich nun ausschliesslich um elektrisehe Reizungen an der Hirnrinde bzw. um Exstirpation von Hirnrindenteilen. Reizungen und Läsionen am 
Zwischenhirn und Mittelhirn sind von Hitzig nicht vorgenommen worden. Nur einmal erwähnt er, dass es ihm gelungen sei, durch Reizung mit einem Lanzenrheophor bei Einstechen in die Hirnsubstanz kombinierte Muskelbewegungen verschiedener Art nachzuweisen. Anfangs war er geneigt, die letzterwähnten Reizeffekte auf die grossen Ganglien zu beziehen. Später hielt er es aber doch für wahrscheinlich, dass nur eine Reizung von nahe bei einander verlaufenden Fasern der inneren Kapsel stattgefunden habe.

Experimentelle Untersuchungen an den basalen Hirnganglien und insbesondere am Thalamus opticus wurden zuerst von Luys, Schiff, Burdach und Magendie vorgenommen. Bej dem von diesen Forschern in Anwendung gezogenen Verfahren handelte es sich aber um sehr grobe mit erheblichen Verletzungen der Nachbarschaft verbundene Eingriffe.

Wirklich auf den Sehhügel beschränkte Läsionen wurden zuerst von Nothnagel ausgeführt und zwar mittels Injektion von Chromsäure mit Hilfe einer Pravaz'schen Spritze, wodurch das betroffene Gewebe gereizt und zugleich zerstört wurde. Der gleichen Methode bediente sich Fournié, jedoch unter Verwendung von Chlorzink anstatt der Chromsäure. Dieser Methode haftete jedoch auch der Nachteil an, dass die Läsion infolge Eindringens der Flüssigkeit in die Umgebung nicht auf den Sehhügel beschränkt blieb. Das erkannte auch Nothnagel bald selbst. Er wandte sich deshalb Versuchen zu, die den $Z_{w e c k}$ hatten, den Sehhügel auf mechanischem Wege zu zerstören. Hierzu benutzte er einen Troikart von der Dicke einer starken Stopfnadel, der nach Eröffnung des Schädels von der Seite her durch einen Teil der Hemisphäre und durch das Ammonshorn in den Thalamus opticus eingeführt wurde. Aus dem freien Ende des Troikarts ragten zwei feine 4 bis $5 \mathrm{~mm}$ lange Federn hervor, die beim Einführen des Instrumentes geschlossen waren und dann so geöffnet werden konnten, dass sie wie die Arme einer Pinzette von einander abstanden. Durch rotierende Bewegungen wurde nun mittels dieser Federn die Läsion im Sehhügel erzeugt und darauf nach Schliessung der Branchen das Instrument wieder herausgezogen. Bei der Obduktion fanden sich umgrenzte Blutherde im Thalamus. Nothnagel selbst hielt es für einen Nachteil, dass diese Blutherde gegenüber den mit Chromsäure erzeugten schon nach einigen Wochen verschwunden waren. Auch betonte er, dass man es natürlich nicht in der Hand habe, den Umfang bezw. die Lage der Läsionen genauer zu bestimmen, dass man vielmehr dabei sehr dem Zufall überlassen sei.

Dieser letztere Umstand bestimmte Ziehen bei seinen im Jahre 1890 veröffentlichten experimentellen Untersuchungen am Sebhügel wieder 
zu dem eingreifenden Verfahren, die Konvexität der Grosshirnhemisphären abzutragen und die grossen Ganglien nach Eröffnung des III. Ventrikels freizulegen. Obwohl er sich nicht verhehlte, dass die basalen Hirnganglien dadurch unter Bedingungen versetzt werden, die von den normalen ganz erheblich abweichen, wählte er diese Versuchsordnung mit Rücksicht darauf, dass nur auf diese Weise eine isolierte Reizung unter Kontrolle des Auges möglich sei. Den freigelegten Thalamus reizte Zi ehen mittels Berührung, Einstich, schwachem faradischem Strom und Schnitt.

Auch eine Reihe von anderen Untersuchern wie Ferrier, Bechterew, Mislawski, Ott und andere sind bei ihren Experimenten in ähnlicher Weise vorgegangen.

Ganz besonders zahlreiche und eingehende Untersuchungen wurden am Thalamus opticus von Probst ausgeführt, deren Resultate in einer Reihe von Arbeiten niedergelegt sind. Den Grund für die weit auseinanderweichenden und vielfach sich direkt widersprechenden physiologischen Ergebnisse der früheren experimentellen Untersuchungen am Thalamus opticus führt Probst auf eine Reihe von Fehlerquellen zurück: es sei keine einheitliche Methode bei den Experimenten angewandt worden, bei den verhältnismässig groben Eingriffen sei die Nachbarschaft des Sehhügels stark in Mitleidenschaft gezogen worden, und vor allem habe eine einwandsfreie Kontrolle der gesetzten Verletzung durch genaue anatomische Untersuchungen der Gehirne an Serienschnitten gefehlt. Es seien also häufig Zerstörungen der Umgebung des Thalamus nicht berücksichtigt, und die physiologischen Folgen dieser Zerstörungen seien ohne genügende anatomisch-histologische Nachuntersuchung dem Sehhügel zugeschrieben worden. Vor allem stellt Probst es wiederholt als eine unerlässliche Forderung auf, dass eine gründliche Untersuchung der Gehirne an lückenlosen Serienschnitten zur exakten Feststellung des Sitzes und der Grösse der Läsion sowie der davon ausgegangenen Degeneration stattfinde, da nur unter dieser Bedingung ein zutreffendes Urteil über die funktionelle Bedeutung der zerstörten Sehhügelgebiete möglich ist. Die genaue anatomische Untersuchung müsse die Grundlage bilden zur Vergleichung der manigfachen von den verschiedenen Untersuchern dem Thalamus zugeschriebenen Funktionen.

Bezüglich der Frage, ob totale Zerstörungen des Sehhügels anzustreben seien, oder ob es ratsamer sei, zirkumskripte Läsionen innerhalb des Thalamus opticus zu setzen, steht Probst auf dem Standpunkt, dass es besser sei, sich mit umschriebenen Läsionen zu begnügen, wenn diese auch nur einen gewissen Teil der Funktion des Sehhügels aufheben, da totale Zerstörungen des Thalamus nicht ohne Mitverletzung der Nachbarschaft $z u$ bewerkstelligen seien. 
Die Läsionen am Sehhügel nahm Probst nach einer von ihm eingefïhrten Methode mit der Hakenkanüle vor, die wohl als eine Verbesserung der Nothnagel'schen Methode bezeichnet werden kann, insofern, als damit isolierte Läsionen des Thalamus ohne Verletzung des Grosshirns, abgesehen rom Balken, gesetzt werden können. Es wird dabei mittels Drillbohrers eine kleine Oeffnung in der Sagittalnaht der Schädelkapsel angebracht, durch welche die Hakenkanüle, welche etwa die Dicke einer Pravaz'schen Injektionskanüle hat, durch den Sulcus interhemisphaericus und durch den Balken hindurch unmittelbar bis in den Sehhügel eingeführt werden kann. Sodann wird ein Haken in beliebiger Länge aus der Kanüle hervorgeschoben, wobei er sich rechtwinklig abbiegt und in den Thalamus eindringt. Durch Emporziehen oder Rotieren des Instrumentes können nun Verletzungen des Sehhügels in vertikaler oder horizontaler Richtung ausgeführt werden. An gehärteten Gehirnen haben die Läsionen das Aussehen von feinen Schnitten.

Probst äussert weiterhin, dass man durch die Kanüle statt des Federhakens auch eine Stromleitung aus feinen Drähten hindurchleiten und aus der Kanüle in den Thalamus vorschieben könne. Auf diese Weise sei es möglich, galvanokaustische Läsionen im Sehhügel herzustellen. Ob Probst diesem Vorschlag entsprechend einmal derartige galvanokaustische Läsionen ausgeführt hat, geht aus seinen Arbeiten, soviel ich sehe, nicht hervor.

Den von früheren Untersuchern wie Ferrier, Ziehen, Bechterew und anderen vorgenommenen elektrischen Reizungen des Sehhügels steht Probst mit grosser Skepsis gegenüber. Als besonders bedenklich hebt er dabei hervor den groben Eingriff, der zur Freilegung des Thalamus zum Zwecke seiner Reizung erforderlich ist, ferner die Unsicherheit, die bestehe betreffs der Reizstelle beim Einführen der Elektroden durch die Grosshirnhemisphären ohne deren Abtragung, und schliesslich den Mangel jeder Kontrolle darüber, wieweit bei den Reizungen Stromschleifen in Frage kommen. Bei Anwendung der elektrischen Reizungen seien daher so lange keine sicheren Resultate zu erwarten, als nicht bessere Methoden für die Reizung gefunden seien.

Eine wesentlich verbesserte Methode der elektrischen Reizung ron in der Tiefe des Gehirns verborgenen Hirnanteilen, wie der basalen Ganglien, ist nun die im Jahre 1904 von Sellier und Verger beschriebene Anwendung von isolierten Nadeln zur Ausführung von elektrischen Reizungen und elektrolytischen Läsionen.

Die gleiche Methode hatten um dieselbe Zeit auch Horsley und Clarke bei ihren Experimenten zur Erforschung des Baues und der 
Funktion des Kleinhirns, unabhängig von den ihnen damals noch nicht bekannten Untersuchungen Sellier's und Verger's, angewandt.

Diese Methode hat vor den früher benutzten mehrere wesentliche Vorzüge :

1. Die Nebenverletzung bei Einführung des Instrumentes zur Anlegung der Läsion beschränkt sich auf ein Minimum, nämlich auf den sehr feinen Stichkanal der isolierten Nadel.

2. Man hat die Möglichkeit, vor Anlegung der Läsion verschiedene Stellen im Bereiche der zu untersuchenden Hirnregion zu reizen und dann die Läsion an einer Stelle anzulegen, die ein besonders wichtiges Reizergebnis geliefert hat.

3. Die Form und Grösse der zu setzenden Läsion lässt sich viel sicherer der Absicht des Untersuchers entsprechend herstellen, als bei allen früheren Methoden. Die Form der Läsionsstelle ist in der Regel annähernd die einer Kugel oder Ellipse in der Umgebung der gereizten Steile und die Grösse derselben verhält sich proportional der Stärke und der Dauer der Stromwirkung.

Diese Methode hat nun durch Horsley und Clarke noch weitere wesentliche Verbesserungen erfahren, welche darauf hinzielten, jede in der Tiefe des Gehirns gelegene Stelle ohne Abtragung des Grosshirns mit möglichster Sicherheit mit dem Pole der Nadel zu erreichen und ferner den elektrischen Reiz auf die Reizstelle selbst zu beschränken, sowie Stromschleifen zu vermeiden.

Zur Erreichung des zuletzt genannten Zieles wird am vorteilhaftesten, wie von Horsley und Clarke ausführlich begründet wurde, eine bipolare Elektrode benutzt, bestehend aus zwei durch feine Glashülsen isolierten Nadeln, deren Spitzen am vorderen Ende aus den Hïlsen hervorragen, aber voneinander durch eine feine Zwischenwand aus Glas geschieden sind. Hierdurch werden die bei Verwendung unipolarer Elektroden infolge von Stromschleifen häufig vorkommenden heftigen Konvulsionen der Tiere, sowie sonstige Nebenwirkungen vermieden.

Ein weiterer ausserordentlich wichtiger Fortschritt gegenüber den früheren Methoden der Anlegung experimenteller Läsionen an tiefliegenden Hirnteilen liegt nun in der Anwendung des von Clarke erfundenen stereotaxischen Instrumentes, welches einerseits jegliche Verschiebung des Kopfes des Tieres gegenüber der Nadel unmöglich macht und somit ein Abbrechen derselben mit Sicherheit verhindert, andererseits es ermöglicht, mit dem Pole der Nadel auf Grund vorheriger genauer Berechnung jeden beliebigen Punkt in der Tiefe des Gehirns auf Millimeternähe genau zu erreichen. 
Zur Ermöglichung dieser Berechnung schufen Horsley und Clarke zunächst eine von der unregelmässigen und schwer zu bestimmenden Oberfläche des Schädels und Gehirns unabhängige kranio-cerebrale Topographie, welche auf der Anlegung dreier auf einander senkrecht stehender Schnittebenen, einer sagittalen, horizontalen und frontalen beruht.

1. Die sagittale Ebene geht in der Richtung der Falx cerebri zwischen den beiden Grosshirnhemisphären hindurch.

2. Die horizontale Ebene verläuft $10 \mathrm{~mm}$ oberhalb und parallel zur basalen Ebene, deren Richtung bestimmt ist durch 4 Punkte, nämlich durch die beiden knöchernen unteren Augenhöhlenränder einer'seits und durch die Zentren der knöchernen Gehörgänge andererseits.

3. Die Frontalebene geht senkrecht zu den beiden ersteren in frontaler Richtung durch die Zentren der knöchernen Gehörgänge.

Durch diese drei Ebenen, die als sagittale, horizontale und frontale Nullebene bezeichnet werden, wird das Gehirn in 8 verschiedene Segmente eingeteilt:

1. Zwei frontale, dorsal ron der horizontalen und oral von der frontalen Schnittebene.

2. Zwei temporale, ventral von der horizontalen und oral von der frontalen Schnittebene.

3. Zwei occipitale, dorsal von der horizontalen und kaudal ron der frontalen Schnittebene.

4. Zwei cerebellare, ventral von der horizontalen und kaudal ron der frontalen Schnittebene.

Jedes von diesen Segmenten bildet also die drei inneren Oberflächen eines Kubus, dessen einzelne Punkte sich von diesen gradlinigen Flächen aus ohne Rücksicht auf die unregelmässig gekrümmte Oberfläche des Gehirns bestimmen lassen.

Diese Segmente sind nun wieder von den Nullebenen aus nach allen drei Dimensionen in parallel verlaufende, je $1 \mathrm{~mm}$ ron einander entfernte, weitere sagittale, horizontale und frontale Schnittebenen eingeteilt, was am einfachsten durch Auflegen einer in Quadratmillimeter eingeteilten Glasplatte zur Darstellung gebracht wird. Auf diese Weise lässt sich also jeder Kubikmillimeter im Inneren des Gehirns, deren das Gehirn rom Macacus Rhesus ungefähr 200000 enthält, genau ermitteln.

Die exakte Lage der Punkte im Inneren des Gehirns, welche gereizt bezw. zerstört werden sollen, lässt sich vor Austührung des Experimentes an Photographien von Gefrierschnitten durch die Köpfe von mittelgrossen Rhesusaffen und Katzen, an welchen die Verbindung der Zentren der 
knöchernen Gehörgänge deutlich sichtbar gemacht ist, ganz genau mit Hilfe eines Zirkels ausmessen. Wenn die Messung genau stimmen soll, so müssen natürlich für die Experimente womöglich Rhesusaffen und Katzen von mittlerer Grösse benutzt werden, deren Köpfe kaum in der Grösse von einander abweichen. Bei Verwendung grösserer oder kleinerer Tiere muss hierauf entsprechend Rücksicht genommen werden. Hunde sind wegen der verschiedenen Form und Grösse ihrer Köpfe weniger zu experimentellen Untersuchungen mit dieser Methode geeignet.

Das stereotaxische Instrument Clarke's besteht aus einem Messingrahmengestell, das namentlich an den knöchernen Gehörgängen nach

Fig. 1.

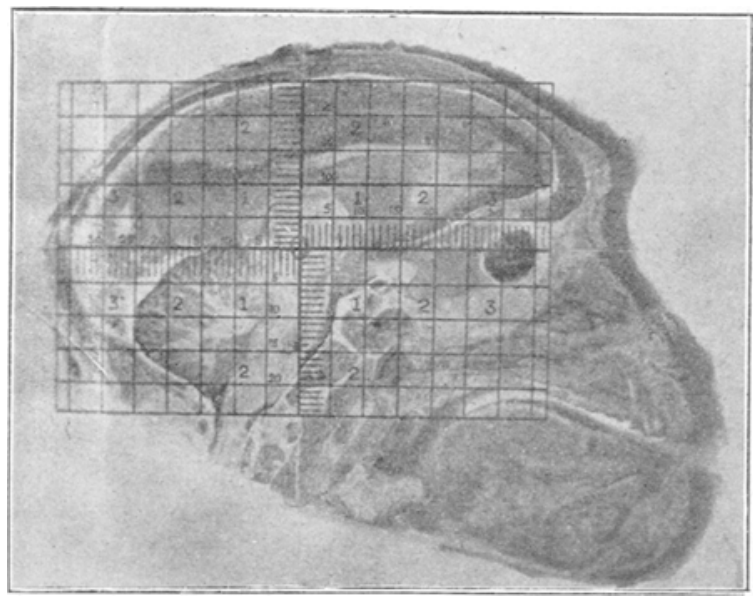

Sagittaler Gefrierschnitt durch den Kopf vom Macacus Rhesus mit Millimeter-(ìasplatte. $\left.{ }^{1}\right)$

vorheriger Einführung von metallenen Ohrpflöcken und am Gesichtsschädel durch Klammern, die von oben gegen den unteren Augenhöhlenrand und von unten her gegen die Alveolen des Oberkiefers anpressen, sowie dureh einige weitere Unterstützungsschrauben absolut sicher am Scliädel befestigt ist, so dass eine Verschiebung des Kopfes gegenüber dem Rahmengestell unmöglich ist. Der Apparat ist so aufgehängt, dass er sich gerade in Höhe des Kopfes des auf den Bauch liegenden Tieres befindet.

Die bipolare Nadel ist am Rahmengestell so angebracht, dass sie sowohl von oben, als auch von vorn und hinten und eventuell auch von

1) Reproduktion einer Abbildung aus der Arbeit von Horsley u. Clarke: The Structure and Functions of the Cerebellum etc. Brain. 1908. Bd. 31. S. 10. 
den Seiten her in den Schädel eingeführt werden kann. Sie gleitet an einer graduierten Messingstange und lässt sich nach allen drei Dimensionen verschieben, so dass jeder vorher an der Photographie des entsprechenden Gefrierschnittes berechnete Punkt im Inneren des Schädels von der Spitze der Nadel erreicht werden kann.

F'ig. 2.

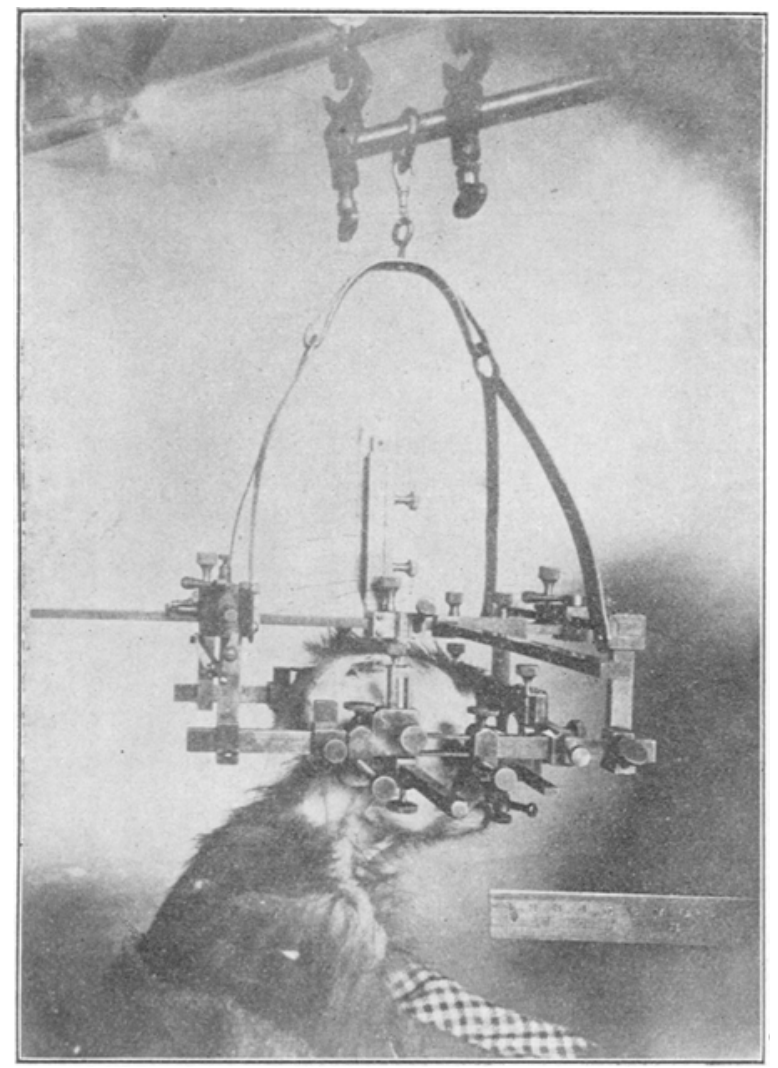

Stereotaxisches Instrument von Clarke am Kopf eines Macacus Rhesus befestigt. Einführung der Nadel von oben.

Der Thalamus opticus liegt bei einem mittelgrossen Macacus Rhesus fast ganz im frontalen und temporalen Segment, mit Ausnahme des hinteren Endes des Pulvinar, welches in das occipitale Segment hineinragt. Beim Einführen der Nadel von oben her kann man auch zwecks Ermittlung des gewünschten Punktes auf der Gefrierschnittphotographie statt der Entfernung desselben oberhalb oder unterhalb von der hori- 
zontalen Nullebene direkt die Entfernung von der Hirnoberfläche aus messen. So wurde z. B. der mediale Thalamuskern bei mittelgrossen Katzen 2-4 $\mathrm{mm}$ seitlich von der Mittellinie, $7-10 \mathrm{~mm}$ oral von der frontalen Nullebene und in einer Tiefe von $16-18 \mathrm{~mm}$ von der Hirnoberfläche gefunden.

Mit Hilfe der beschriebenen Methode gelang es zunächst Horsley und $\mathrm{Clarke}$ selbst, in einer Reihe von Experimenten die Kleinhirnkerne exakt zu reizen bezw. zu zerstören.

Sodann hat E. Sachs im Laboratorium von Horsley an einem ausgedehnten Tiermaterial von Rhesusaffen und Katzen Reizungen und Läsionen am Thalamus opticus vorgenommen und ist dabei zu bemerkenswerten Resultaten bezüglich der Funktion des Sehhügels und seiner Faserverbindungen gelangt. Die von E. Sachs am Thalamus opticus gesetzten elektrolytischen Läsionen waren aber von verhältnismässig geringem Umfang. Hierauf führte er es selbst zurück, dass er keine abwärts degenerierten Fasern nach dem roten Kern und nach weiter kaudal gelegenen Teilen des Zentralnervensystems nachweisen konnte.

Ich selbst habe nun im Jahre 1911 ebenfalls in Horsley's Laboratorium für experimentelle Neurologie mit Benützung des von Horsley und Clarke ausgearbeiteten Verfahrens experimentelle Untersuchungen am Zwischen- und Mittelhirn ausgeführt. Dabei wurden aber zur sicheren Erzielung sekundärer Degeneration grössere Läsionen als die von Sachs gesetzten angelegt. Die Läsionen nahmen am Sehhügel in der Regel einen ganzen Kern ein und gingen oft auch noch auf benachbarte Kerne über.

Im ganzen wurden an 45 Tieren, nämlich an 10 Rhesus-Affen und an 35 Katzen Experimente ausgeführt, die zum Teil von Horsley, zum Teil von mir selbst vorgenommen wurden.

Bei sämtlichen Tieren wurden zunächst unter Chloroformäthernarkose nach Anlegung einer Trepanationsöffnung an der linken Schädelseite mittels der bipolaren Nadel faradische Reizungen im Bereich des Thalamus opticus, des Hypothalamus und der Vierhügelgegend ausgeführt, nachdem vorher die zu reizende Stelle mit Hilfe der Gefrierschnittsphotographien genau ausgerechnet war. Dabei wurde der faradische Strom, gemessen nach Kronecker-Einheiten (zwischen 500 und 10000) zunächst von kurzer und, wenn hierbei kein Reizeffekt zu erzielen war, von längerer Dauer angewandt. Die Nadel wurde bei den Affen in der Regel vom Hinterhaupt aus in horizontaler Richtung durch das Kleinhirn hindurch zum Zwecke der Vermeidung einer Balkenverletzung eingeführt, bei den Katzen dagegen, bei welchen dies wegen der knöchernen Beschaffenheit des Tentoriums nicht ausführbar ist, stets senkrecht von oben nach unten. 
Nach Vornahme der faradischen Reizungen wurde an einer Stelle, die auf Grund eines bemerkenswerten Reizeffektes besonders geeignet erschien, eine elektrolytische Läsion gesetzt. Hierbei wurde der galvanische Strom in der Regel von 5-10 M. A. Stärke und 10 Minuten Dauer angewandt.

Die Tiere wurden jeweils mindestens 14 Tage am Leben erhalten, während dieser Zeit genau beobachtet und in Bezug auf das Verhalten der Motilität, der Sensibilität, des Sehrermögens, der Pupillen- und Sehnenreflexe untersucht. Namentlich wurde auf abnorme Körperhaltung und etwaige Abänderung des Ganges geachtet. Nach Ablauf der Beobachtungszeit wurde dann bei den Tieren noch das elektrische Verhalten der Grosshirnrinde bezw. der Kleinhirnkerne und der Vierhügel durch faradische Reizungen in Narkose geprïft zwecks Feststellung etwaiger infolge der Läsion eingetretener Veränderungen. Dann wurden die Tiere durch vermehrte Chloroformdosis getötet.

Das Gehirn wurde sodann nach mehrtägiger Behandlung in FormolMüller'scher Lösung in der Richtung der mit Hilfe des stereotaxischen Apparates markierten frontalen Null-Ebene durchschnitten und darauf parallel zu dieser Schnittebene in $2 \mathrm{~mm}$ dicke Frontalschnitte zerlegt, die, nachdem photographische Aufnahmen der Läsionen gemacht waren, nach der Marchi'schen Methode weiter behandelt wurden. Schliesslich wurden lückenlose Frontalserien sämtlicher Gehirne angefertigt.

Von den 45 Tieren, an welchen Experimente vorgenommen wurden, gelang es, 29 mindestens 14 Tage am Leben zu erhalten, deren Gehirne dann in der soeben beschriebenen Weise weiter behandelt und untersucht wurden. Unter den zur weiteren Untersuchung verwendeten Tieren handelte es sich 19 mal um reine Thalamusläsion. Bei den übrigen waren ausserdem die Regio subthalamica und die Vierhügel von der Läsion mitbetroffen.

Auf die anatomischen und physiologischen Resultate der Untersuchungen soll hier nicht näher eingegangen werden. Die Veröffentlichung derselben bleibt späteren Mitteilungen unter Beifügung der Protokolle und photographischen Abbildungen vorbehalten. Hier soll es sich nur um eine einleitende Erörterung technischer Fragen handeln, die zugleich dazu dienen soll, das von Horsley und Clarke ausgearbeitete Verfahren zur experimentellen Untersuchung in der Tiefe verborgener Hirnteile, das alle anderen Untersuchungsmethoden auf diesem Gebiete weit übertrifft, und das, soweit mir bekannt, in Deutschland noch keine weitere Anwendung gefunden hat, zu allgemeinerer Kenntnis zu bringen.

Der eingangs gegebene historische Riickblick zeigt, dass diese Methode alle von dem Altmeister Hitzig in Bezug auf experimentelle Unter- 
suchungen am Gehirn überhaupt, sowie auch die von Probst in Bezug auf den Thalamus opticus gestellten Forderungen erfüllt und zugleich die Nachteile der früher angewandten Methoden vermeidet.

Die Methode ermöglicht vor allem, ohne nennenswerte Nebenverletzung beliebige in der Tiefe des Gehirns gelegene Stellen nach vorausgegangener genauer Berechnung zu reizen und dann an der Reizstelle eine Läsion von beliebiger Grösse anzulegen. Frïher war, um den Sehhügel mit Sicherheit elektrisch zu reizen, zuvor ein grober Eingriff nötig, um ihn freizulegen; wurde dieser Eingriff vermieden, und die Elektrode direkt durch die Hemisphäre hindurch geführt, so blieb es stets im höchsten Grade unsicher, ob tatsächlich der Sehhïgel gereizt war oder die Nachbarschaft. Bei dem Horsley'schen Verfahren ist aber die Reizstelle durch die an gleicher Stelle gesetzte elektrolytische Läsion genau markiert. Die Anwendung der bipolaren Nadel verhindert das Eintreten von Stromschleifen und gestattet so die Auffindung der Foci, wobei es zugleich möglich ist, durch schwache und kurzdauernde faradische Ströme isolierte Reizerscheinungen, durch stärkere und länger dauernde kombinierte Muskelbewegungen auszulösen. Besonders geeignet ist das Verfahren zur Anlegung der von Hitzig geforderten, kleinen, den Reizstellen entsprechenden Läsionen. Auch können infolge der vorhergehenden Berechnung an der Hand der Gefrierschnittsphotographien, sowie infolge der genauen Einstellung der Nadel mit Hilfe des stereotaxischen Instruments gleichwertige Versuche beliebig oft wiederholt werden, und die einzelnen Versuche können so beschrieben werden, dass jederzeit ein Vergleich der von verschiedenen Untersuchern vorgenommenen Experimente angestellt werden kann.

Infolge der Möglichkeit einer genanen Berechnung der zu lädierenden Stelle sind Verletzungen der Nachbarschaft eine grosse Seltenheit, wie sich auch bei meinen Resultaten gezeigt hat.

Probst hat zwar mit seiner Hakenkanüle auch zirkumskripte Läsionen des Thalamus erzielt, sein Verfahren hat aber den Nachteil, dass es keine elektrischen Reizungen erlaubt, sondern dass mit dem von ihm geübten Einschnitt sofort auch Ausfallserscheinungen auftreten müssen. Ein weiterer Uebelstand seiner Methode ist auch, dass jedes Mal von der medialen Seite in den Sehhügel eingegangen werden muss, dass also unter allen Umständen der mediale Thalamusanteil verletzt wird, so dass niemals isolierte Läsionen des vorderen, lateralen und ventralen Thalamuskerns oder des Pulvinars zustande gebracht werden können. 


\section{Literaturverzeichnis.}

1. Bechterew, Virchow's Archiv. 1887. Bd. 110. S. 252.

2. Bechterew, Neurol. Zentralbl. 1894. Bd. 13. S. 252.

3. Bechterew, Neurol. Zentralbl. 1897. Bd. 16. S. 145.

4. Bechterew, Neurol. Zentralbl. 1905. Bd. 25. S. 545.

5. Bechterew, Monatsschr. f. Psych. and Neurol. 1905. Bd. 27. S. 224.

6. Bechterew und Mislawski, Neurol. Zentralbl. 1888. Bd. 7. S. 505.

7. Bechterew und Mislawski, Neurol. Zentralbl. 1891. Bd. 10. S. 481.

8. Burdach, Bau und Leben des Gehirns. Leipzig 1819.

9. Ferrier, Functions of the brain. London 1886.

10. Fournié, Recherches sur les fonctions du cerveau. Paris 1873.

11. Goltz, Ueber die Verrichtungen des Grosshirns. Bonn 1881.

12. Hitzig und Fritsch, Ueber elektrische Erregbarkeit des Grosshirns. Archiv f. Physiol. 1870.

13. Hitzig, Alte und neue Untersuchungen über das Gehirn. Archir f. Psych. Bd. 34,35 und 36 .

14. Horsley and Clarke, The Structure and Funktions of the Cerebellum examined by a new method. Brain 1908. Vol. 31. p. 1.

15. Luys, Sur le système nerveux. Paris 1855.

16. Magendie, Le système nerveux. Paris 1841 .

17. Munk, Ueber die Ausdehnung der Sinnessphären in der Grosshirnrinde. Sitzungsberichte. 1900. S. 782 .

18. Nothnagel, Untersuchungen am Gehirn. Virchow's Archiv. 1874. Bd. 52.

19. Ott, Vaso-tonic Centres in the Thalami. Journ. of nervous and mental diseases. 1891. Vol: 17. p. 483.

20. Probst, Deutsche Zeitschr. f. Nervenheilk. 1898. Bd. 13. S. 384.

21. Probst, Deutsche Zeitschr. f. Nervenheilk. 1900. Bd. 17. S. 141.

22. Probst, Monatsschr. f. Psych. u. Neurol. 1900. Bd. 7. S. 387.

23. Probst, Monatsschr. f. Psych. u. Neurol, 1900. Bd. 8. S. 165.

24. Probst, Monatsschr. f. Psych. u. Neurol. 1901. Bd. 10. S. 288.

25. Probst, Archiv f. Psych. 1900. Bd. 33. S. 721.

26. Probst, Archiv f. Psych. 1902. Bd. 35. S. 22.

27. Sachs, On the Structure and Funktional Relations of the optic Thalamus. Vol. 32. p. 95.

28. Sellier et Verger, Lésions de la couche optique. Compt. rend. Soc. de Biol. 1898. Vol. 5. p. 522.

29. Sellier et Verger, Idem. 1903. p. 485.

30. Zi ehen, Zur Physiologie der intrakortikalen Ganglien usw. Arch. f. Psych. 1890. Bd. 21. 